\title{
Adherence to an Occupational Blood Borne Pathogens Exposure Management Program Among Healthcare Workers and Other Groups at Risk in Argentina
}

\author{
Marisa Miceli ${ }^{1}$, Fabián Herrera ${ }^{1}$, Elena Temporiti ${ }^{1}$, \\ Dong $\mathrm{Li}^{2}$, Andrea Vila ${ }^{1}$ and Pablo Bonvehí ${ }^{1}$
}

\author{
Division of Infectious Diseases, Department of Internal \\ Medicine, CEMIC'; Buenos Aires, Argentina; Myeloma \\ Institute for Research and Therapy, University of Arkansas \\ for Medical Sciences ${ }^{2}$, Little Rock, Arkansas, USA
}

\begin{abstract}
We conducted a retrospective review of 130 occupational blood borne pathogens exposure (BBP-OE) records at Centro de Estudios Médicos e Investigaciones Clínicas, a university hospital with an ongoing educational program and a postexposure management program for healthcare workers (HCWs) since 1995, in order to evaluate adherence to a hospital BBP-OE management program. We compared HCWs from our institution (Group 1) and HCWs from independent institutions that contract our postexposure management program (Group 2). Compliance with standard precautions in Group 1 was inadequate in $77 \%, 23 \%$, and $16 \%$ of nurses, physicians, and others, respectively. A greater proportion of HCWs in Group 1 (74\% vs. 40\%) reported occupational accidents within two hours after exposure $(p=0.0001)$. No difference was observed regarding compliance with adherence to schedule, partial adherence, and loss at follow-up (14\%, $33 \%$, and $53 \% ; p>0.05$ ). Adherence to the standard of care for BBP-OE, including postexposure prophylaxis, was low (HIV: $53 \%$ and HBV: $63 \%$ ). Knowledge of the seropositive status of the source patient did not improve adherence. We conclude that postexposure programs do not guarantee appropriate behavior by $\mathrm{HCWs}$. General interventions and ongoing personnel education to modify individual attitudes are needed, as are continued efforts to assess HCWs' experiences with these programs, as well as the identification of strategies to improve adherence.

Key Words: Healthcare workers, postexposure prophylaxis.
\end{abstract}

Hepatitis B virus (HBV), human immunodeficiency virus, (HIV) and hepatitis $\mathrm{C}$ virus (HCV) infections constitute well-recognized occupational risks for healthcare workers (HCWs). Avoiding occupational blood exposure is a cornerstone of preventing transmission of these blood-borne pathogens (BBP) in the hospital setting [1,2]. During the past 15 years, the U.S. Public Health Service and the Centers for Disease Control and Prevention (CDC) have published guidelines for the management of occupational blood Received on 07 August 2005; revised 17 November 2005. Address for correspondence: Dr. Marisa Miceli. Research Associate, Myeloma Institute for Research and Therapy, University of Arkansas for Medical Science. 4301 West Markham \#776, Little Rock, AK 72205, USA. Phone: (501) 296-1503 ext 1553. Fax: (501)686-6442.E-mail: micelimarisah@uams.edu

The Brazilian Journal of Infectious Diseases 2005;9(6):454-458 (C) 2005 by The Brazilian Journal of Infectious Diseases and Contexto Publishing. All rights reserved. exposure [1,3]. These recommendations have become the standard of care for occupational exposure to BBP.

A hospital's occupational postexposure management program should encourage prompt reporting, evaluation, and counseling, and should provide prophylactic drugs and follow-up [2]. A program of transmission prevention should provide educational programs for $\mathrm{HCW}$ s that emphasize the importance of implementing standard precautions (SP). It should also instruct HCWs how to proceed in the event of an occupational exposure potentially involving a BBP.

It is well recognized that the effectiveness of these strategies depends on the compliance and adherence to a program by healthcare-facility personnel. However, there is growing concern about how well occupational-exposure-management programs are accepted and about the range of adherence to 
postexposure prophylaxis (PEP), hepatitis B vaccination (HBVv), and to follow-up by $\mathrm{HCW}$ s after initial assessment [4-7].

We examined the epidemiology of occupational exposures and of adherence to our hospital's occupational BBP exposure management program.

\section{Material and Methods}

This descriptive study was conducted at CEMIC (Centro de Estudios Médicos e Investigaciones Clínicas), a university hospital with an infectious diseases department and an occupational medicine clinic, which has maintained an ongoing $\mathrm{HCW}$ educational program (which provides up-to-date information and instructions regarding infection control, postexposure prophylaxis, prompt reporting, and serological testing after exposure) and a postexposure management program since 1995 . These programs also provide up-to-date information regarding the use of SP. Interactive courses are given to new employees, and twice-a-year refresher courses are mandatory for established employees. Pamphlets and newsletters are widely available in all hospital areas.

We reviewed the medical records of all HCWs with occupational exposures who had been followed at our institution between June 1995 and October 1999. Two groups were identified: Group 1 consisted of all HCWs from CEMIC; Group 2 consisted of HCWs of other independent institutions that contract our postexposure management program.

The timing of the exposure report, source-patient BBP serological data, $\mathrm{HCW}$ serologic status at the time of exposure and follow-up serological data were recorded. Data regarding occupational Groups (Group 1 or Group 2), route of exposure, SP implementation at exposure, compliance to postexposure prophylaxis (PEP and HBVv), and adherence to follow-up, were collected and analyzed. An HBV surface antibody was tested for in all HCWs at baseline, and an HBV vaccination was administered as required [1].

Physicians in the emergency department in our hospital provide immediate, standardized postexposure assistance 24 hours a day, year round. Exposure data are collected using specially designed medical records, and a blood sample for HBV, HCV, and HIV screening is drawn immediately in the event of possible exposure to a BBP. Infectious disease specialists provide 24hour consultation coverage for clinical guidance to the emergency staff, counseling for the exposed HCW, and further follow-up. Our hospital's postexposure policies are derived from updated CDC recommendations [1]. The hospital provides safety devices for personal protection, appropriate handling of body fluids, and disposal of sharp instruments. Availability of on-site serological testing, first-line antiretroviral postexposure prophylaxis drugs, HBV gamma globulin, and infectioncontrol resources are guaranteed at our institution 24 hours a day.

Standard precautions were defined as the first line of defense in infection control; SP assume that all blood and body fluids are potentially infectious. They include diligent hygiene practices (i.e., hand-washing and drying), use of personal protective equipment (i.e., gloves, gowns, masks, and eye protection), and appropriate handling and disposal of sharp instruments (i.e., safe transfer, no needle recapping, immediate disposal after use). Standard precautions are used when handling nonintact skin, mucous membranes, blood, and all other body fluids, even if dried.

Four different types of adherence were defined:

1. Adherence to SP was defined as an HCW's detailed description of the procedure performed (e.g., needle-stick, splashes, etc) at the time of exposure. Data regarding SP and the use of safety devices were collected.

2. Adherence was defined as an HCW who attended $100 \%$ of the follow-up visits on the dates the appointments had been scheduled.

3. Partial adherence was defined as an HCW attending fewer than $100 \%$ of the scheduled follow-up appointments and/or an HCW who attended the follow-up visits on a different date than the appointments had been scheduled.

4. Loss to follow-up was defined as an HCW who did not attend the scheduled follow-up appointments. 


\section{$\underline{\text { Statistical analysis }}$}

We evaluated the frequencies of the four different types of adherence (as defined above) to our hospital's BBP-OE management program. SAS 8.2 (SAS Institute Inc, Cary, NC) software was used for the analyses, which consisted of a two-tailed chi-square $\left(\chi^{2}\right)$ test for differences in contingency table probabilities. Statistical significance was defined as $p$ $<0.05$.

\section{Results}

We reviewed 130 reported HCW exposures that occurred between June 1995 and October 1999, divided into two groups: 80 from CEMIC (Group 1) and 50 from other institutions (Group 2).

Occupational groups of exposed HCWs included $51(39 \%)$ nurses, $22(17 \%)$ physicians, $8(6 \%)$ technicians, $6(5 \%)$ phlebotomists, $8(6 \%)$ scrub nurses, $2(2 \%)$ medical students, and $33(25 \%)$ housekeepers and others (doormen, sanitation workers, and caretakers). One hundred and two (78\%) of the reported exposures were due to needle-stick and scalpel injuries. Other exposures included $6(5 \%)$ splashes and $13(10 \%)$ others (skin injuries due to glass and/or plastic materials in contact with body fluids). Nine $(7 \%)$ were unknown (data not available). Seventy-nine HCWs $(61 \%)$ reported the exposure within the first two hours.

Overall $27(21 \%)$ of the source patients were seropositive for HIV, HCV, and/or HBV (Table 1). Compliance with SP among HCWs in Group 1 was inadequate in $77 \%, 23 \%$, and $16 \%$ of nurses, physicians, and others. A greater proportion of HCWs in Group 1 (74\% vs. 40\%) reported occupational accidents within two hours after exposure $(p=0.0001)$.

However, no significant differences were observed regarding compliance with adherence, partial adherence, and lost to follow-up, when both groups were compared (Table 2; $\mathrm{p}>0.05$ ).

The proportions of HCWs classified as loss to followup, partial adherence, or adherence were 53\%, 33\%, and $14 \%$. This rate was $56 \%, 26 \%$, and $18 \%$ among 27 HCWs exposed to a known seropositive source. Knowing the seropositive status of source patients did not improve adherence to postexposure management.

Adherence to PEP(HIV and HBV) was not as good as was expected. PEP for HIV was prescribed in 7 HCWs; only 4 (57\%) completed it. The HBVv was indicated in $28 \mathrm{HCW}$, and only $19(68 \%)$ completed the schedule.

\section{Discussion}

We assessed the epidemiology of occupational exposures to BBP among HCWs and adherence to our hospital's occupational BBP exposure management program. In agreement with previous publications [810], nurses and housekeeping personnel were the mostexposed groups of HCWs. This may be because nurses and housekeeping personnel comprise the largest group of HCWs. Similarly, we found that needle sticks were the most frequent route, as previously reported in other studies $[9,10]$.

The number of occupational accidents reported within two hours after exposure was significantly higher among HCWs from our institution $(p=0.0001)$. This supports previous published data suggesting that SP training improves self-reporting of BBP exposure [7]. However, there was remarkably poor adherence to the follow-up schedule in our population (only 14\%), especially considering that this is a key part of our program; our HCWs routinely receive a telegram reminding them of the next scheduled appointment.

One would think that knowing the infectious-disease status of the source patient, as well as understanding the risks of transmission, might make HCWs more conscientious about adherence to infectious-diseases prescriptions. However, in our study, adherence to follow-up by HCWs who had been exposed to seropositive source patients was lower than expected, even when source patients were seropositive for HIV, $\mathrm{HBV}$, or HCV. Our experience suggests that availability of resources and information is not enough to guarantee HCW's' compliance. 
Table 1. Serological status of source patients in cases of exposure accidents

\begin{tabular}{lcc}
\hline & $\mathbf{N}$ & $\mathbf{\%}$ \\
\hline Tested source & 101 & 78 \\
Source patient positive for Hepatitis C virus antibody & 13 & \\
Source patient positive for Hepatitis B virus surface antigen & 5 & \\
$\quad$ Source patient positive for Hepatitis C virus antibody & 4 & \\
$\quad$ and for Hepatitis B virus surface antigen & 5 & \\
$\quad$ Source patient with a positive ELISA test for HIV & - & - \\
Seroconversion among exposed HCWs & 29 & 22 \\
Untested source & & \\
\hline
\end{tabular}

$\mathrm{HCW}=$ Healthcare workers.

Table 2. Adherence to an occupational blood borne pathogens exposure management program among healthcare workers in Argentina

\begin{tabular}{|c|c|c|c|c|c|}
\hline & Management program & \multicolumn{2}{|l|}{ Total } & \multirow{2}{*}{$\begin{array}{c}\chi^{2} \\
\text { test statistics }\end{array}$} & \multirow[t]{2}{*}{ P value } \\
\hline & $\begin{array}{l}\text { Group 1 } \\
(\mathbf{n}=\mathbf{8 0})\end{array}$ & $\begin{array}{l}\text { Group } 2 \\
(\mathbf{n}=\mathbf{5 0})\end{array}$ & $(n=130)$ & & \\
\hline $\begin{array}{l}\text { First report } \\
(<2 \text { hours })^{(a)}\end{array}$ & $57(74)$ & $20(40)$ & 79 (61) & 14.7012 & $0.0001 * * *$ \\
\hline Adherence & $14(18.5)$ & $4(8)$ & $18(14)$ & 2.3279 & 0.1271 \\
\hline Partial adherence $^{(\mathbf{b})}$ & $23(29)$ & $20(40)$ & $43(33)$ & 1.7592 & 0.1847 \\
\hline Lost to follow-up & $43(54)$ & $26(52)$ & $69(53)$ & 0.0378 & 0.8458 \\
\hline
\end{tabular}

***significant at $\mathrm{a}=0.05$.Percentages are given in parentheses. (a) Accident reported within the first two hours after exposure. (b) Partial adherence: adherence to follow-up not according to established schedule.

Limitations of the current study include underreporting, dependence on care-provider analysis, and retrospective chart review. Adherence was analyzed from the healthcare provider's point of view, and no data were obtained regarding HCWs' reasons for noncompliance to follow-up (e.g., emotional stress following an occupational exposure might be an important variable to consider when evaluating a program like this, as this information might be helpful in designing a program that better fits HCWs' needs).

There is great diversity in the educational and cultural backgrounds among HCWs in developing countries such as Argentina. For example, the coexistence of professional (graduated from University) with nonprofessional nurses (including "auxiliary nurses" graduated from non-university nursing schools and a minority of "vocational nurses" who had learned their skills but did not attend any of the previously mentioned institutions) working in the same hospital is not uncommon. This phenomenon, which is extremely rare in a university hospital, is more frequently seen in outlying regions. We suspect that this diversity among HCWs might vastly affect the rate of adherence to a given hospital's program and should be considered in its design. Although it cannot be determined from our study, we mention it here as a potential variable for future studies.

Additionally, populations at risk for occupational exposure to $\mathrm{HIV}, \mathrm{HBV}$, and $\mathrm{HCV}$ are constantly 
changing. An increasing number of groups are considered at risk of BP exposure, including police officers [11]. In our study, caretakers, doormen, and sanitation workers were among the groups exposed to BBP. Thus, these groups, as well as members of the general population, should be aware of the risks of BBP, avoid exposure to BBP, and should seek prompt medical assistance when exposure does occur.

We conclude that although training does improve self-reporting of BBP exposure, the availability of resources and postexposure programs do not guarantee appropriate HCW behavior (such as adherence to SP, PEP and follow up) in hospitals. We believe that structured interventions and ongoing personnel education targeted at modifying individual attitudes are still needed. Further efforts are also needed to assess HCWs' experiences with hospital programs and to identify strategies to improve adherence.

\section{References}

1. Beltrami E.M., Alvarado-Ramy F., Critchley S.E., et al. Updated U.S. Public Health Service Guidelines for the Management of Occupational Exposures to HBV, HCV and HIV and Recommendations for Postexposure Prophylaxis. MMWR 2001;50(RR-11):1-52.

2. Henderson D.K. HIV postexposure prophylaxis in the $21 \mathrm{st}$ century. Emerg Infect Dis 2001;7(2):254-8.

3. Kessler H.A., Presbyterian R. HIV Post-exposure Prophylaxis (PEP): Rationale and Recommendations. In: Clinical Infectious Diseases, editor. 39th Annual Meeting of the Infectious Diseases Sociaty of America; 1999; Philadelphia; 1999:S86.

4. Moloughney B.W. Transmission and postexposure management of bloodborne virus infections in the health care setting: where are we now? CMAJ 2001;165(4):445-51.

5. Garb J.R. One-year study of occupational human immunodeficiency virus postexposure prophylaxis. J Occup Environ Med 2002;44(3):265-70.

6. Fahey B.J., Koziol D.E., Banks S.M., Henderson D.K. Frequency of nonparenteral occupational exposures to blood and body fluids before and after universal precautions training. Am J Med 1991;90(2):145-53.

7. Wang S.A., Panlilio A.L., Doi P.A., et al. Experience of healthcare workers taking postexposure prophylaxis after occupational HIV exposures: findings of the HIV Postexposure Prophylaxis Registry. Infect Control Hosp Epidemiol 2000;21(12):780-5.
8. Behrman A.J., Shofer F.S., Green-McKenzie J. Trends in bloodborne pathogen exposure and follow-up at an urban teaching hospital: 1987 to 1997 . J Occup Environ Med 2001;43(4):370-6.

9. Gershon R.R., Flanagan P.A., Karkashian C., et al. Health care workers' experience with postexposure management of bloodborne pathogen exposures: a pilot study. Am J Infect Control 2000; 28(6):421-8.

10. Sanchez M., Del Rio C., Whittkamp M., et al. Poor Acceptance and Tolerability of Post Exposure prophylaxis (PEP) Following Occupational Exposure Among Health Care Workers (HCW) at an Ubarn Medical Center. In: Clinical Infectious Diseases, editor. 37th Annual Meeting of the Infectious Diseases Society of America; 1999; Philadelphia, p. 390.

11. McGuinn M., Smith K., Sha B., et al. Occupational HIV risk and Post Exposure Prophylaxis in Chicago Police Officers. In: Clinical Infectious Diseases, editor. 39th Annual Meeting of the Infectious Diseases Society of America; 2001; San Francisco; 2001:167. 